OPEN ACCESS

Edited by:

Keyu Xie,

Northwestern Polytechnical

University, China

Reviewed by:

Edward Gillan,

The University of lowa, United States

Sung Mook Choi,

Korea Institute of Materials Science,

South Korea

*Correspondence:

Ye Wang

wangye@zzu.edu.cn

Specialty section:

This article was submitted to

Electrochemistry,

a section of the journal

Frontiers in Chemistry

Received: 07 July 2019 Accepted: 09 October 2019

Published: 25 October 2019

Citation:

Yan C, Xu T, Ma C, Zang J, Xu J, Shi Y, Kong D, Ke C, Li X and Wang Y

(2019) Dendrite-Free Li Metal

Plating/Stripping Onto

Three-Dimensional

Vertical-Graphene@Carbon-Cloth

Host. Front. Chem. 7:714.

doi: 10.3389/fchem.2019.00714

\section{Dendrite-Free Li Metal Plating/Stripping Onto Three-Dimensional Vertical-Graphene@Carbon-Cloth Host}

\author{
Congcong Yan ${ }^{1}$, Tingting $\mathrm{Xu}{ }^{1}$, Caiyun $\mathrm{Ma}^{1}$, Jinhao Zang ${ }^{1}$, Junmin $\mathrm{Xu}{ }^{1}$, Yumeng Shi ${ }^{2}$, \\ Dezhi Kong ${ }^{1}$, Chang $\mathrm{Ke}^{3}$, Xinjian $\mathrm{Li}^{1}$ and Ye Wang ${ }^{1 *}$ \\ ${ }^{1}$ Key Laboratory of Material Physics of Ministry of Education, School of Physics and Engineering, Zhengzhou University, \\ Zhengzhou, China, ${ }^{2}$ International Collaborative Laboratory of 2D Materials for Optoelectronics Science and Technology of \\ Ministry of Education, Institute of Microscale Optoelectronics, Shenzhen University, Shenzhen, China, ${ }^{3}$ School of Electrical \\ and Electronic Engineering, Nanyang Technological University, Singapore, Singapore
}

Lithium metal is deemed as an ideal anode material for next-generation lithium ion batteries (LIBs) due to its high specific capacity and low redox potential. However, uncontrolled lithium dendrite formation during electrochemical deposition leads to a low Coulombic efficiency and serious safety issues, dragging metallic lithium anodes out of practical application. One promising strategy to suppress lithium dendrite issues is employing a three-dimensional host with admirable conductivity and large surface area. Herein, a vertical graphene nanosheet grown on carbon cloth (VG/CC) synthesized is adopted as the $\mathrm{Li}$ deposition host. The three-dimensional VG/CC with a large surface area can provide abundant active nucleation sites and effectively reduce the current density, leading to homogeneous Li deposition to overcome the dendrite issue. The Li@VG/CC anode exhibits a dendrite-free morphology after a long cycle and superior electrochemical performance to that of planar $\mathrm{Cu}$ current collector. It delivers a small voltage hysteresis of $90.9 \mathrm{mV}$ at a high current density of $10 \mathrm{~mA} \mathrm{~cm} \mathrm{~cm}^{-2}$ and a Coulombic efficiency of $99 \%$ over 100 cycles at $2 \mathrm{~mA} \mathrm{~cm}{ }^{-2}$. Our results indicate that this all-carbon-based nanostructure host has great potential for next-generation $\mathrm{Li}$ metal batteries.

Keywords: Li metal anode, three-dimensional VG/CC, dendrite-free, excellent electrochemical performance, long cycle stability

\section{INTRODUCTION}

Lithium-ion batteries (LIBs) have dominated the energy storage market owing to its high energy density, long cycle life, absence of memory effect and low pollution (Chu and Majumdar, 2012; Chu et al., 2016; Cheng et al., 2017; Lim et al., 2019a). However, the energy density of currently commercial LIBs needs to be further improved (Du et al., 2016). One of the solutions is exploring high-capacity anode materials, as the theoretical capacity of commercial graphite is only $372 \mathrm{mAh}$ $\mathrm{g}^{-1}$ (Bruce et al., 2012; Cheng et al., 2016b). Among various advanced anode materials, lithium metal has a high theoretical capacity $\left(3,860 \mathrm{mAh} \mathrm{g}^{-1}\right)$ and low electrochemical potential $(-3.04 \mathrm{~V}$ 
vs. the standard hydrogen electrode; Cheng et al., 2016a). Therefore, lithium metal is considered to be one of the most promising anode materials (Xu et al., 2014; Zhang et al., 2016). In particular, lithium metal is also widely employed as the anode of other high-energy density batteries such as Li-S and $\mathrm{Li}-\mathrm{O}_{2}$ batteries (Cheng et al., 2015; Grande et al., 2015; Ma et al., 2015; Manthiram et al., 2015; Huang et al., 2018). Therefore, it is timely and urgent to develop high performance lithium metal anode (Huang et al., 2014). However, the formation of unstable solid electrolyte interphase (SEI) due to lithium metal high electrochemical reactivity and infinite volume change upon repeated plating/stripping causes the severe uncontrolled lithium dendrite growth, leading to a low Coulombic efficiency and internal short circuit even severe safety issue (Lim et al., 2019b).

In order to tackle these issues, great efforts have been devoted to depress the lithium dendrite formation: optimization of the electrolytes with additives, artificial SEI layer, nanoscale interface engineering, etc. (Liu B. et al., 2018). A 3D electrode host with a large surface area is demonstrated as an effective strategy to suppress the dendrite formation due to the reduced current density (Li et al., 2017; Ma et al., in press). Graphene is a kind of carbon materials with a single/few layers of carbon atoms arranged in a hexagonal lattice (Deng et al., 2014; Lin et al., 2016). As a two-dimensional material with many merits, horizontal graphene has been used as the acritical SEI layer and 3D host of lithium metal anode (Chen et al., 2018; Liu S. et al., 2018; Wu et al., 2018; Li et al., 2019). Different from the traditional graphene grown/stacked in the horizontal direction, vertical graphene $(V G)$ is a type of graphene grown in vertical direction, naturally hosting the lithium metal. For example, 3D vertical graphene nanowalls (VGN) grown on nickel (Ni) foam (VGN/Ni) by mesoplasma chemical vapor deposition (MPCVD) are employed as the lithium metal host (Ren et al., 2018). The symmetric battery VGN/Ni@Li stably cycles more than 2,000 h at a current density of $0.5 \mathrm{~mA} \mathrm{~cm}^{-2}$ (Ren et al., 2018). Vertical graphene nanosheets grown on copper foam (Cu@VG) prepared by plasma-enhanced chemical vapor deposition (PECVD) is also employed as the lithium metal host (Hu et al., 2019). The assembledCu@VG@Li battery can stably cycle for 100h at a current density of $3 \mathrm{~mA} \mathrm{~cm}{ }^{-2}$ with a capacity of $3 \mathrm{mAh} \mathrm{cm}^{-2}(\mathrm{Hu}$ et al., 2019). VG on $\mathrm{Ni} / \mathrm{Cu}$ foam has a large surface area, which provides plenty of nucleation centers for the lithium metal and effectively reduces the current density, resulting in an improved electrochemical performance. All the previous reports employed metal ( $\mathrm{Ni}$ or $\mathrm{Cu}$ ) as the scaffold to support the graphene. Compared with $\mathrm{Ni}$ or $\mathrm{Cu}$ foam, carbon scaffold has the merits of being lightweight, flexible, low-cost and renewable (Xu et al., 2017; Li et al., 2018b; Jiang et al., 2019). Carbon-based VG host is of great interest for flexible, low-cost and sustainable lithium metal batteries. However, there have been no reports of carbon as the scaffold to support VG as the lithium metal host until now.

Herein, we report a metal-free 3D interconnected VG grown on the surface of carbon cloth (VG/CC) as the host of lithium metal to form a dendrite-free nanostructure. With the porous $3 \mathrm{D}$ structure, VG/CC allows controllable and dendrite-free lithium metal plating/stripping (Figure 1). The volume change during the plating/stripping process is also well-adapted (Wang et al., 2018b). The three-dimensional structure increases the specific surface area relative to the planar $\mathrm{Cu}$, reducing the local current density, effectively inhibiting dendrite growth, and improves long-term cycle stability and rate performance. The battery can stably cycle with a long lifespan over 500 cycles at the current density $10 \mathrm{~mA} \mathrm{~cm}^{-2}$ and a high Coulombic efficiency of $>99 \%$. In order to explore the real application, a full battery composed of Li@VG/CC as the anode and the $\mathrm{LiCoO}_{2}$ (LCO) as the cathode exhibits a capacity of $133 \mathrm{mAh} \mathrm{g}^{-1}$ at $0.2 \mathrm{C}$.

\section{MATERIALS AND METHODS}

\section{Synthesis of VG/CC}

The synthesis process of the VG/CC can be found in previous reports (Wang et al., 2016; Ghosh et al., 2017). In brief, VG grown on CC was synthesized by a plasma-enhanced chemical vapor deposition (PECVD) and CC as substrate was placed in the center of the plasma-generating region. The atmosphere was $\mathrm{CH}_{4} / \mathrm{H}_{2}$ (40/60\%), the pressure was controlled to $25 \mathrm{~Pa}$ and the RF power was $1,000 \mathrm{~W}$. The growth was $20 \mathrm{~min}$ to synthesis VG/CC with a substrate temperature of $700^{\circ} \mathrm{C}$.

\section{Materials Characterizations}

The morphology of the as-prepared VG/CC was characterized by field emission scanning electron microscopy (JEOL, JSM$6700 \mathrm{~F}$ ) and high-resolution transmission electron microscopy (HRTEM, JEOL, JEM-2100). The crystal structure of the sample was performed by X-ray diffraction with $\mathrm{Cu} \mathrm{K} \alpha$ radiation (XRD SmartLab $3 \mathrm{KW}$ ). Raman spectra were carried out by confocal Raman spectroscopy (HORIBA, LabRAM HR Evolution).

\section{Electrochemical Deposition to Fabricate Li@VG/CC Anode and Electrochemical Performance Evaluation}

The Li@VG/CC was fabricated by a standard electrochemical deposition process. In brief, the prepared VG/CC and the lithium foil were used as the working and counter electrode, respectively. Two pieces of membrane (Celgard 2400) were used as the separator, and $1 \mathrm{M}$ lithium bis(trifluoromethane)sulfonylimide (LiTFSI) in 1,3-dioxolane: 1,2-dimethoxyethane binary solvent (DOL:DME, $1: 1$ by volume) with $1 \% \mathrm{LiNO}_{3}$ (stabilizer) was used as the electrolyte. All of the above components were assembled into CR2032-type coin cells in an argon-filled glove box $(<0.1$ ppm of oxygen and water) for the next step of the electrochemical deposition process. The metallic Li was plated onto the substrate of VG/CC and planar $\mathrm{Cu}$ by a Neware battery discharge/charge equipment. All cells were cycling at $0-1 \mathrm{~V}$ (vs. $\mathrm{Li}^{+} / \mathrm{Li}$ ) at $50 \mu \mathrm{A}$ five times to stabilize SEI film and remove surface contamination. For the Coulombic efficiency measurement, 1 or $2 \mathrm{mAh} \mathrm{cm}^{-2}$ capacity was plated on VG/CC and then recharged to $1 \mathrm{~V}$ at current densities of 1 or $2 \mathrm{~mA} \mathrm{~cm}^{-2}$. The Coulombic efficiency was evaluated by the stripping-capacity/plating-capacity. For long-term constant current discharge/charge tests, all batteries were plated with a constant current capacity of $1 \mathrm{mAh} \mathrm{cm}^{-2}$ and stripped up to $1 \mathrm{~V}$ at a current density of 1,2 , and $10 \mathrm{~mA} \mathrm{~cm}^{-2}$. Electrochemical impedance spectroscopy (EIS) was measured by an electrochemical workstation (VMP3, Bio-logic) after various 


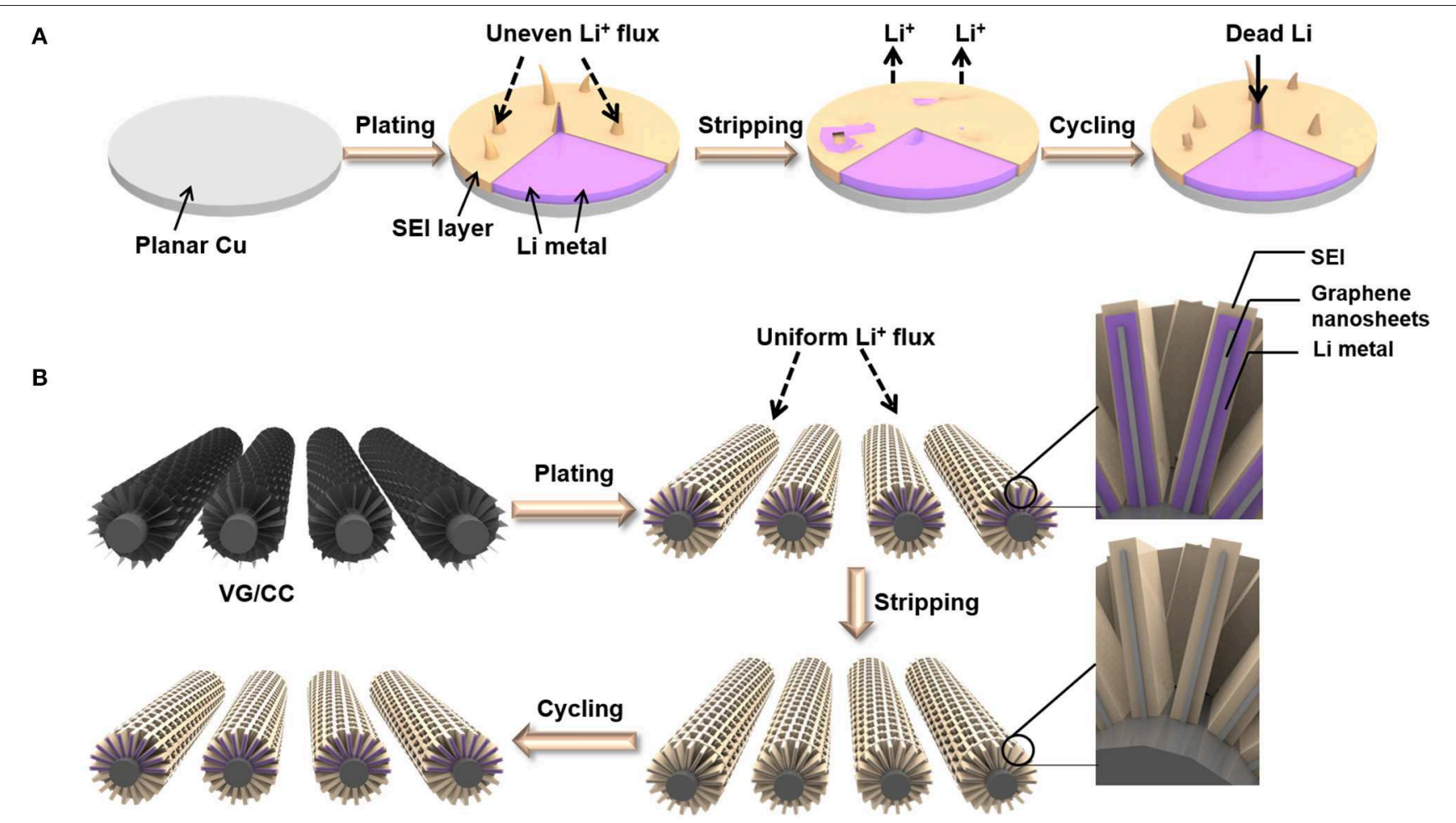

FIGURE 1 | Schematic illustration of the Li plating/stripping process on (A) planar Cu and (B) VG/CC.

electrochemical cycles by applying an alternating voltage of $10 \mathrm{mV}$ over the frequency ranging from $10^{5}$ to $10^{-2} \mathrm{~Hz}$. The control sample was Li deposited onto a piece of two-dimensional planar $\mathrm{Cu}$, all deposition processes and evaluation processes were the same as that of Li@VG/CC.

For full battery testing, commercial $\mathrm{LiCoO}_{2}$ was used as the positive electrode. A total of $80 \% \mathrm{LiCoO}_{2}, 10 \%$ polyvinylidene fluoride (PVDF), and 10\% carbon black were thoroughly ground in air until being homogeneously mixed. Then, NMP was added dropwise to form the slurry, then coated onto an $\mathrm{Al}$ foil. The resulting electrode sheet contains $\sim 2 \mathrm{mg} \mathrm{cm}^{-2}$ of $\mathrm{LiCoO}_{2}$. For full battery long-term cycling, $2 \mathrm{mAh} \mathrm{cm}^{-2} \mathrm{Li}$ metal was deposited onto VG/CC or planar $\mathrm{Cu}$ to match the $\mathrm{LiCoO}_{2}$. The electrolyte was $1 \mathrm{M} \mathrm{LiPF}_{6}$ in ethylene carbonate (EC) and diethyl carbonate (DEC) (1:1 by volume). The voltage test range was $2.5-4.5 \mathrm{~V}$.

\section{RESULTS AND DISCUSSIONS}

The synthesis process of VG/CC is described in detail in Figure 2a. Vertically grown graphene nanosheets on the surface of the CC are synthesized by a PECVD (Ghosh et al., 2017). CC consists of interconnected carbon nanofibers with good flexibility, strong mechanical strength and high electron transport capability (Figure S1; Tian et al., 2018a). However, CC is lithiophibic with limited surface area (Liu F. et al., 2019). After PECVD process, VG nanosheets are uniformly grown on the surface of CC (Figures $\mathbf{2 b}-\mathbf{d}$ ). The synthesized VG/CC exhibits flexible property as shown in the inset of Figure $\mathbf{2 b}$. The VG grown on the vertical direction may be attributed to the localized electrical field and the plasma/reaction process (Wang et al., 2016). There are plenty of pores/voids between the VG nanosheets, leading to the increased surface area compared to the planar $\mathrm{Cu}$ (Figure S2). A tip of $\mathrm{VG}$ is characterized by transmission electron microscopy (TEM) (Figures 2e,f). The interplanar spacing of the sample is $0.34 \mathrm{~nm}$, corresponding to the (002) crystal planes of graphene as shown in Figure 2g.

The crystal structure is performed by $\mathrm{X}$-ray diffraction (XRD) as shown in Figure 3A. There are two peaks centered at 26 and $42^{\circ}$, corresponding to (002) and (101) planes of the graphitic carbon, respectively (Huang et al., 2019). Raman spectra of the samples are shown in Figure 3B. There are three peaks located at $1,350,1,590$, and $2,690 \mathrm{~cm}^{-1}$, which can be attributed to $\mathrm{D}, \mathrm{G}$ and $2 \mathrm{D}$ peaks of graphene, respectively (Cho et al., 2018; Meng et al., 2019). The D and G peaks are corresponding to the disorder carbon atoms in the hexagonal graphitic network and the in-plane vibrational mode of $\mathrm{sp2-}$ bonded carbon atoms, respectively (Zhang et al., 2018; $\mathrm{Hu}$ et al., 2019). Thus, the $\mathrm{D}$ peak indicates there are plenty of defects in the VG/CC nanostructures, which are the nucleation sites for lithium metal plating according to a previous study (Wang et al., 2017). Introduced defects in graphene reduce the lithium metal nucleation energy barrier (Tian et al., 2017; Jiang et al., 2019). In addition, a strong $G$ peak and a high 

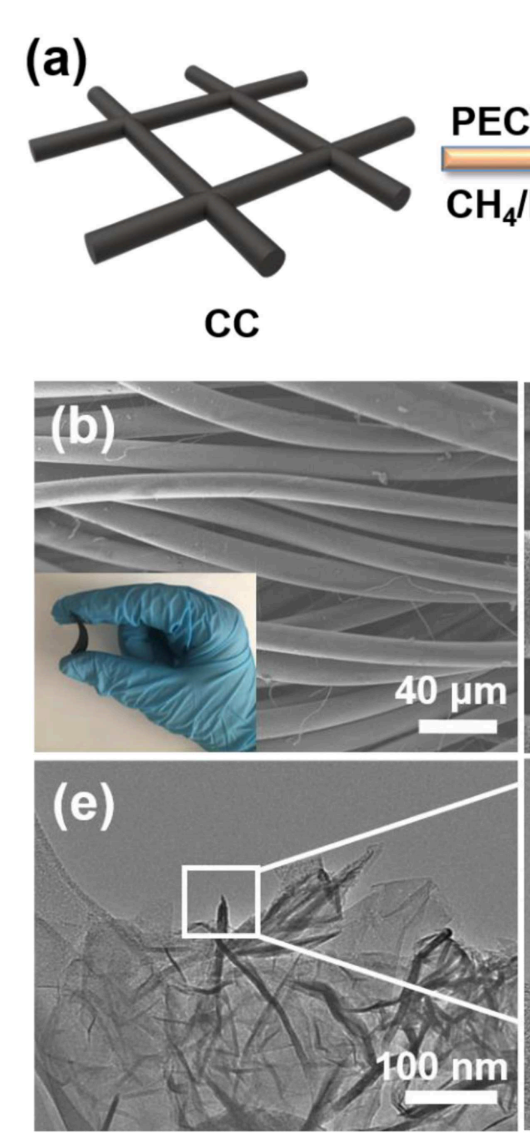

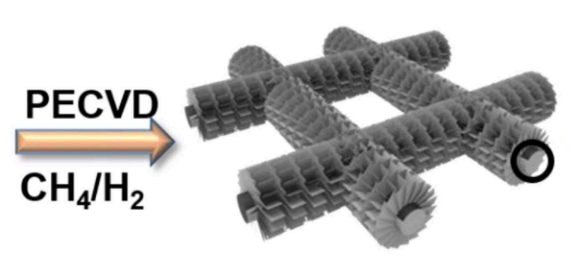

VG/CC
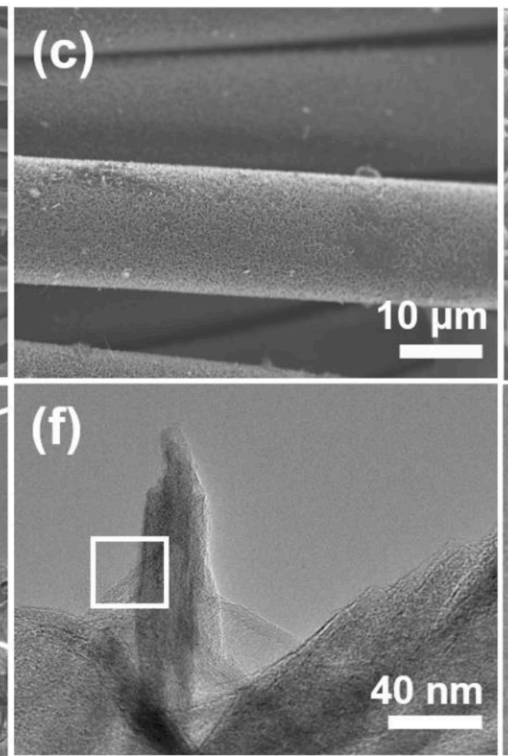
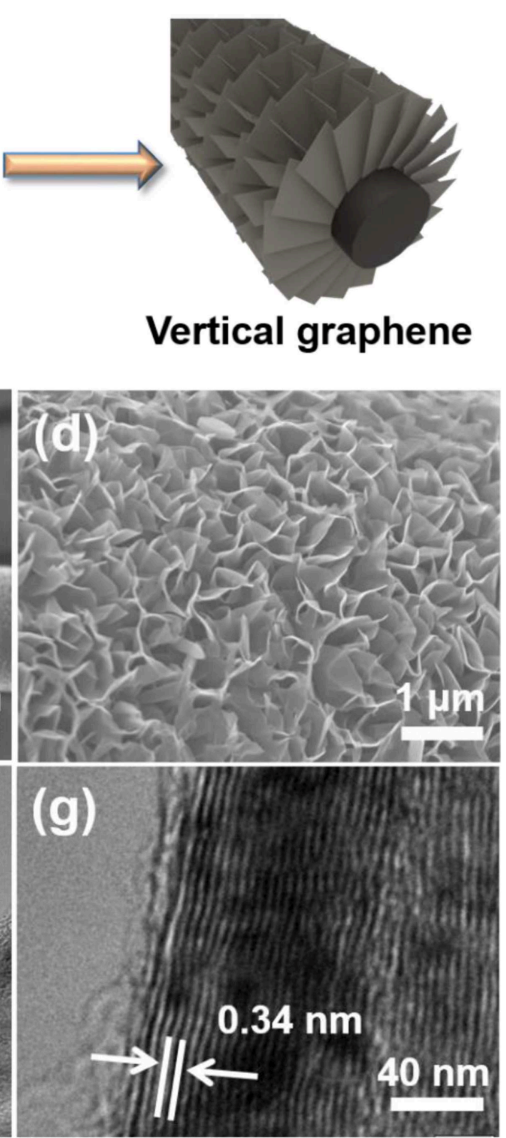

FIGURE 2 | (a) Schematic illustration of the synthesis process of the VG/CC by PECVD. (b) Low, (c) high, and (d) higher magnification SEM images of VG/CC. Insets: photograph of bent VG/CC, demonstrating the good mechanical strength of VG/CC. TEM image of VG/CC at (e) low and (f) high magnifications, (g) HRTEM images of VG/CC.
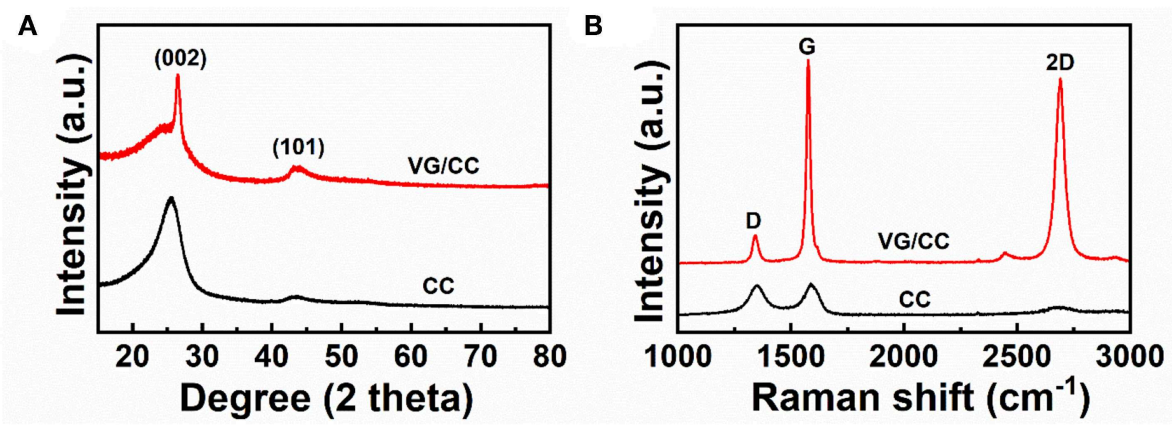

FIGURE 3 | (A) XRD patterns and (B) Raman spectra of the VG/CC and CC.

$I_{G} / I_{D}$ (6.78) indicate that the graphene sheets have a high degree of graphitization with good electronic conductivity (Kong et al., 2019).

In order to investigate the plating/stripping behavior of lithium metal on VG/CC, an electrochemical deposition cell is assembled with VG/CC as the working electrode to electrodeposit lithium metal and lithium foil as the counter electrode to provide the lithium source. The morphology evolution of the VG/CC during the Li metal plating/stripping process is performed by an ex-situ SEM method. As shown in Figure 4a, when initial plated capacity reached up to $0.2 \mathrm{mAh} \mathrm{cm} \mathrm{cm}^{-2}$, metallic lithium is nucleated and uniformly distributed on VG sheets without obvious protuberance. From the corresponding higher magnification SEM image (Figure 4g), 


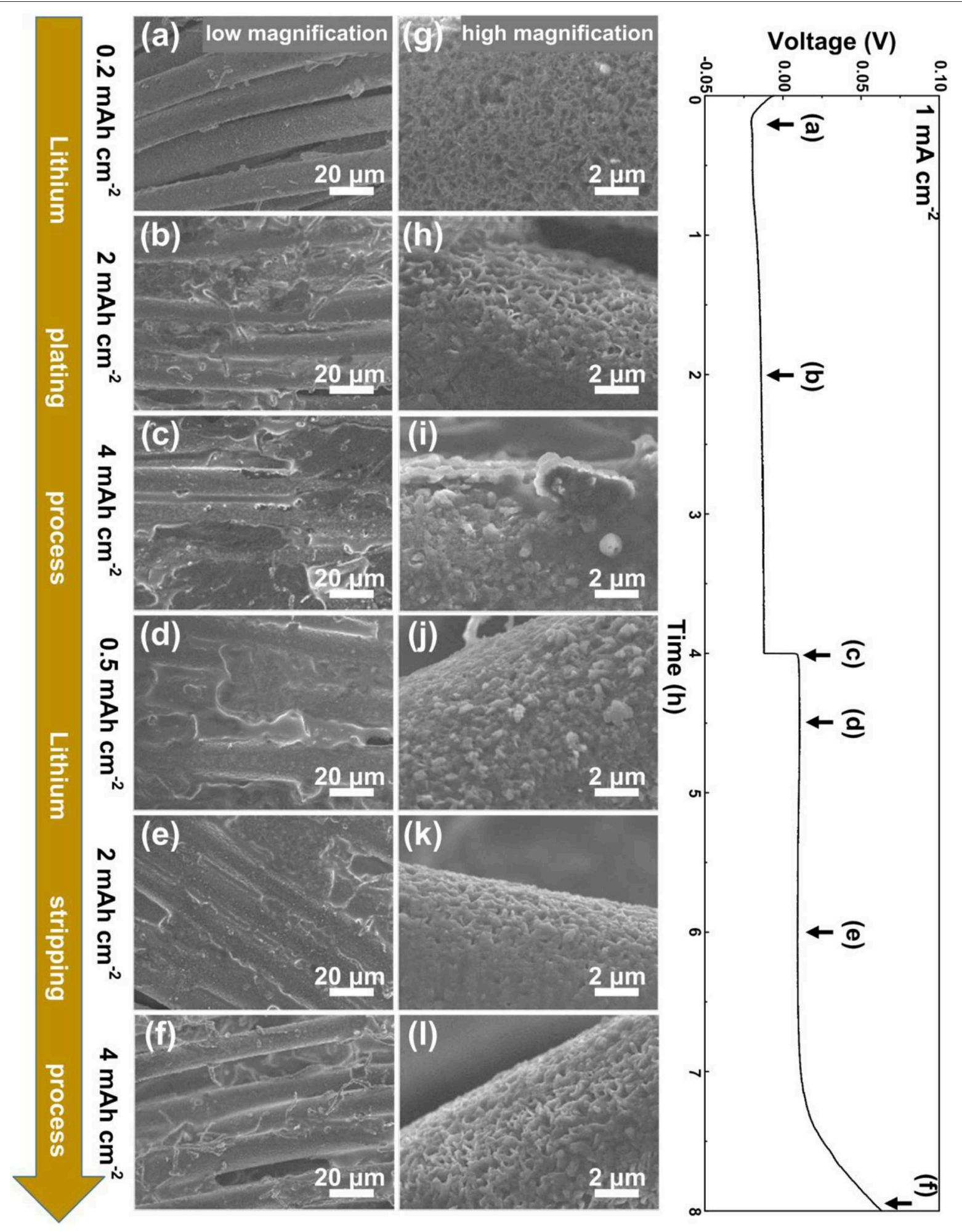

FIGURE 4 | Morphological evolution of Li metal plating and stripping on VG/CC. SEM images of VG/CC electrode after being plated at a capacity of (a,g) $0.2 \mathrm{mAh}$ $\mathrm{cm}^{-2} \mathbf{( b , h )} 2 \mathrm{mAh} \mathrm{cm}^{-2}$, and (c,i) $4 \mathrm{mAh} \mathrm{cm}{ }^{-2}$, and then stripped (d,j) $0.5 \mathrm{mAh} \mathrm{cm}^{-2}$, (e, $\left.\mathbf{k}\right) 2 \mathrm{mAh} \mathrm{cm}^{-2}$, and (f,l) $4 \mathrm{mAh} \mathrm{cm}^{-2}$ at a current density of $1 \mathrm{~mA} \mathrm{~cm}-2$.

the vertically distributed graphene nanosheets can still be clearly seen. With the capacity increased to $2 \mathrm{mAh} \mathrm{cm}^{-2}$ (Figure $4 \mathbf{b}$ ), the VG nanosheets are still visible and the deposited lithium metal fills the pores and starts to cover the VG surface (Figure 4h). It is also noted that the plated Li metal is prone to horizontally expand from the VG nanosheets to the micro-scale voids between the 
A

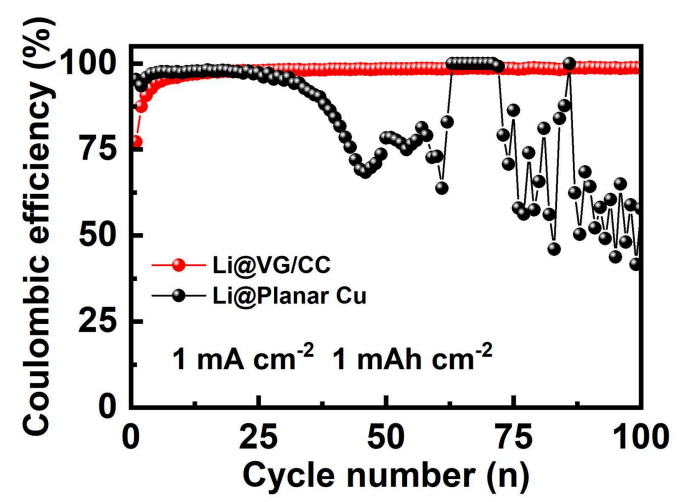

C

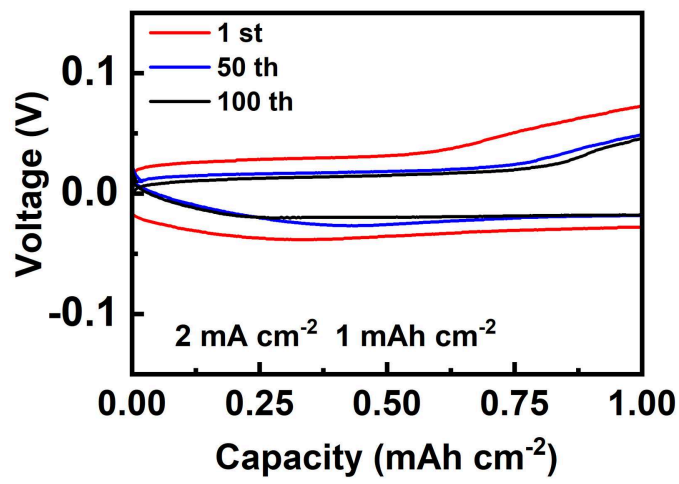

B

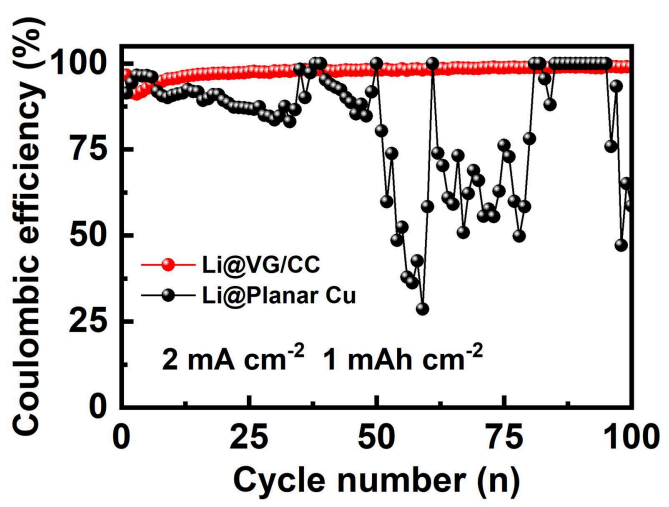

D

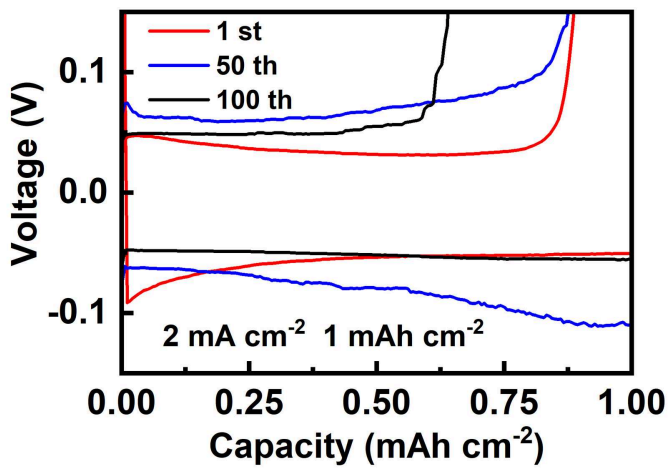

FIGURE 5 | Coulombic efficiency of the VG/CC (red), planar Cu (black) with a cycling capacity of $1 \mathrm{mAh} \mathrm{cm}^{-2}$ at a current density of (A) $1 \mathrm{~mA} \mathrm{~cm}^{-2}$ and (B) $2 \mathrm{~mA}$ $\mathrm{cm}^{-2}$. Voltage-capacity curves of the (C) VG/CC and (D) planar Cu after various cycles with a capacity of $1 \mathrm{mAh} \mathrm{cm}^{-2}$ at a current density of $2 \mathrm{~mA} \mathrm{~cm}^{-2}$.

carbon fibers. This may be owing to the confinement effect due to the nanosheets-reinforced nanostructure (Hao et al., 2016). The excess lithium metal is held in the pores of VG/CC structures. The same phenomena have been observed in nanowire- $\mathrm{Cu}$ foam as the Na metal host (Wang et al., 2018a). With further plating capacity increased to $4 \mathrm{mAh} \mathrm{cm}^{-2}$ (Figure 4c), the graphene nanosheets are almost fully filled with metallic lithium (Figure 4i). Uniformly small size lithium deposits without dendrites are observed, indicating a uniform lithium ion plating process. Moreover, the thickness of VG/CC electrode is increased from $400.00 \mu \mathrm{m}$ (before) to $412.50 \mu \mathrm{m}$ (after Li deposition

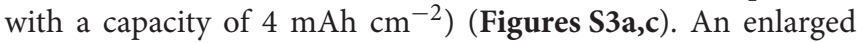
view to show the thickness of VG is changed from $993.75 \mathrm{~nm}$ (before) to $1,312.50 \mathrm{~nm}$ (after Li deposition with a capacity of $4 \mathrm{mAh} \mathrm{cm}^{-2}$ ) (Figures S3b,d). When the lithium is gradually stripped from VG/CC, the lithium metal in pores and voids starts to fade and the VG nanosheets are gradually exposed. When stripping capacity reaches to $0.5 \mathrm{mAh} \mathrm{cm}^{-2}$ (Figure 4d), lithium metal begins to fade but still fully covers the surface of the sample (Figure $\mathbf{4 j}$ ). With the stripping capacity reaching 2 $\mathrm{mAh} \mathrm{cm}{ }^{-2}$ (Figure 4e), and the graphene nanosheets start to be exposed due to the fade of lithium metal (Figure 4k). With further stripping up to $4 \mathrm{mAh} \mathrm{cm}^{-2}$ (Figure 4f), the potential reaches up to $64 \mathrm{mV}$, and the graphene nanosheets are completely revealed (Figure 41), suggesting the high reversibility of VG/CC. In contrast, the plating/stripping behavior of planar $\mathrm{Cu}$ is shown in Figure S4. When plating capacity reaches to $0.5 \mathrm{mAh} \mathrm{cm}^{-2}$, small lithium particles start to nucleate on $\mathrm{Cu}$ foil. With the plating capacity increasing from 0.5 to $4 \mathrm{mAh} \mathrm{cm}^{-2}$, metallic lithium particles grow bigger and longer, and finally grow into moss tree-like lithium dendrites with a diameter of $\sim 10 \mu \mathrm{m}$ (Figure S4i). During the stripping process, the lithium dendrite gradually becomes smaller. When the stripping capacity reaches $3.9 \mathrm{mAh} \mathrm{cm}^{-2}$, the potential is increased to $500 \mathrm{mV}$, the reason is owing to all electrically connected lithium is fully stripped. Therefore, $0.1 \mathrm{mAh} \mathrm{cm}^{-2}$ lithium is irreversible. However, plenty of microdots (indicated by the red circles) and films can be found on the surface of copper foil, corresponding to the dead lithium dendrite and the SEI film (Cheng et al., 2018; Li et al., 2018a).

Coulombic efficiency is a critical parameter to evaluate the reversibility of the metallic lithium plated into the host. In our work, Coulombic efficiency is measured at different current densities 1 and $2 \mathrm{~mA} \mathrm{~cm}^{-2}$ as shown in Figure 5. The Coulombic efficiency of VG/CC is increased in the first several cycles and then stabled $>99 \%$ after 20 cycles at 1 and $2 \mathrm{~mA} \mathrm{~cm}^{-2}$. However, the Coulombic efficiency of planar $\mathrm{Cu}$ first decreased quickly and fluctuated at all measured conditions. For example, at a capacity of $1 \mathrm{mAh} \mathrm{cm}^{-2}$, the Coulombic efficiency of planar $\mathrm{Cu}$ is higher than $98 \%$ at a current density of $1 \mathrm{~mA} \mathrm{~cm}-2$ from the 1 st to 25 th cycle, and then dropped quickly and fluctuated (Figure 5A). It is more unstable at a current density of $2 \mathrm{~mA} \mathrm{~cm}^{-2}$ with the capacity of $1 \mathrm{mAh} \mathrm{cm}^{-2}$ (Figure $5 \mathrm{~B}$ ). 
In comparison, the Coulombic efficiency of VG/CC gradually stable at $99 \%$ at current densities of $1 \mathrm{~mA}$ and $2 \mathrm{~mA} \mathrm{~cm}^{-2}$ (both at $1 \mathrm{mAh} \mathrm{cm}^{-2}$ ). It is worth mentioning that the Coulombic efficiency of VG/CC is also higher than $97 \%$ at $1 \mathrm{~mA} \mathrm{~cm}{ }^{-2}(2$ $\mathrm{mAh} \mathrm{cm}^{-2}$ ) (Figure S5). The inferior Coulombic efficiency of the planar $\mathrm{Cu}$ is due to the uneven $\mathrm{Li}$ nucleation and the dendrite growth induced unstable SEI film (Lin et al., 2017). The reason for the Coulombic efficiency of VG/CC at initial several cycles is lower than $99 \%$ is due to the irreversible lithium that is consumed to form the SEI layer (Adams et al., 2018). After stable SEI film is formed, the Coulombic efficiency is increased higher than
$99 \%$. Benefiting from the large surface conductive area inducing reduced current density and the plentiful nucleation centers, the dendrites are inhibited by the designed VG/CC lithium host. The Li plating and stripping curves at various cycles of VG/CC and planar $\mathrm{Cu}$ are shown in Figures $5 \mathrm{C}, \mathrm{D}$, respectively. The voltage hysteresis of VG/CC is $67.3,44.5$, and $35.1 \mathrm{mV}$ at 1 , 50 , and 100 cycles, respectively. For comparison, the voltage hysteresis of planar $\mathrm{Cu}$ is $85.3,148.8$, and $100.4 \mathrm{mV}$ at 1,50 , and 100 cycles, respectively. Small voltage hysteresis indicates excellent plating/stripping behavior. Overpotential is another key parameter to evaluate the electrochemical performance of

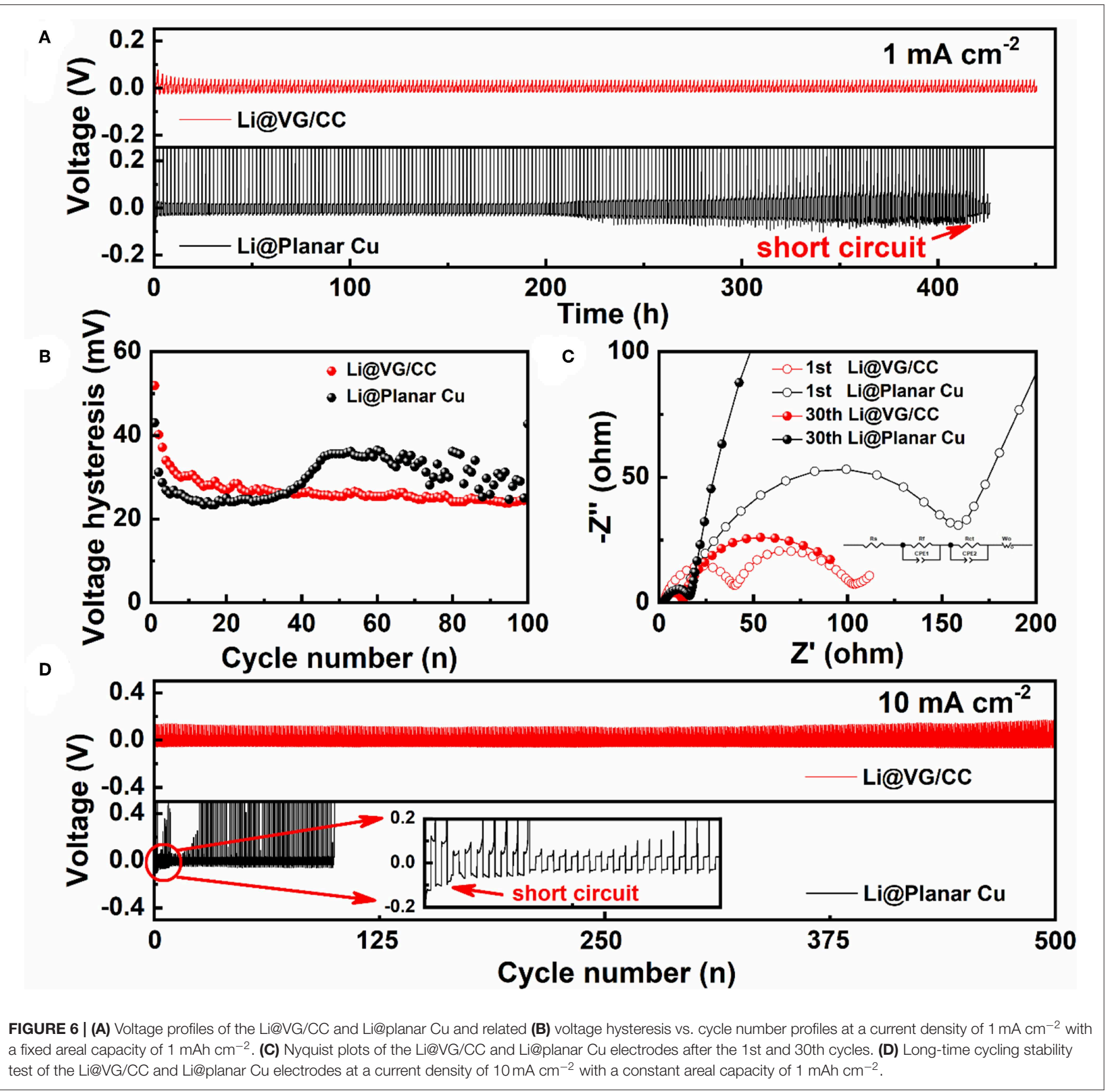


TABLE 1 | Comparison of the performance of the VG/CC and other 3D Li metal hosts.

\begin{tabular}{|c|c|c|c|c|}
\hline & Current density $(\mathrm{mA} \mathrm{cm}-2)$ & Area capacity $\left(\mathrm{mAh} \mathrm{cm}^{-2}\right)$ & Cycle number & References \\
\hline VG/CC & 10 & 1 & 500 & This work \\
\hline Graphitized carbon fibers & 2 & 1 & 300 & Zuo et al., 2017 \\
\hline Cufoam @VG & 3 & 3 & 50 & Hu et al., 2019 \\
\hline 3D porous $\mathrm{Cu}$ & 2 & 1 & 120 & Li et al., 2017 \\
\hline N doped GCF & 3 & 1 & 900 & Liu L. et al., 2018 \\
\hline 3D ALD-CNTS & 3 & 1 & 90 & Zhang et al., 2017 \\
\hline CC/CNT & 5 & 1 & 1,250 & Liu F. et al., 2019 \\
\hline Oriented graphene foam (OGF) & 10 & 0.5 & 250 & Ma et al., 2018 \\
\hline Oxygen-CNT & 4 & 1 & 200 & Liu K. et al., 2018 \\
\hline Vertical grapheme nanowalls on Ni foam & 0.5 & 1 & 500 & Ren et al., 2018 \\
\hline
\end{tabular}

A

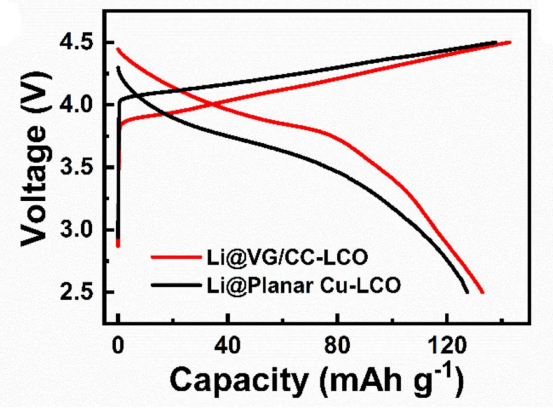

B

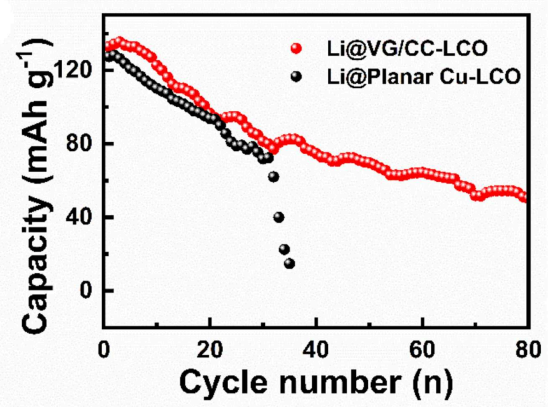

FIGURE 7 | Electrochemical performance of a full battery with Li@VG/CC as the anode and LCO as the cathode: (A) charge-discharge curves at a current density of $0.2 \mathrm{C}\left(1 \mathrm{C}=372 \mathrm{mAh} \mathrm{g}^{-1}\right)$ in a potential range of $2.5-4.5 \mathrm{~V}$, and $(\mathbf{B})$ cycling performance at $0.2 \mathrm{C}$.

the lithium metal anode (Liu Y. et al., 2019). The nucleation overpotential is the difference between the lowest voltage point (nucleation potential) during discharge and the voltage platform after stabilization, which is used to overcome heterogeneous nucleation (Yan et al., 2016). The nucleation overpotential of VG/CC is $44.3 \mathrm{mV}$, which is much smaller than that of planar $\mathrm{Cu}(101.6 \mathrm{mV})$ in Figure S6. The lower overpotential means that lithium metal is more easily nucleated on the host. It is worth mentioning that the $\mathrm{Li}$ ions start to insert into the VG/CC above $0 \mathrm{~V}$ (Tian et al., 2018a). When the voltage continuous decreased below $0 \mathrm{~V}$ until the nucleation potential (indicated by the circle in Figure S6), the metallic lithium starts to nucleate and grow in the format of lithium metal (Tian et al., 2018b).

The electrochemical behavior of the Li@VG/CC and Li@planar $\mathrm{Cu}$ is evaluated by measuring their long-term cycling stability. The planar $\mathrm{Cu}$ and $\mathrm{VG} / \mathrm{CC}$ are repeated plating/stripping at a fixed area capacity of $1 \mathrm{mAh} \mathrm{cm}^{-2}$ at a current density of $1 \mathrm{~mA} \mathrm{~cm}^{-2}$ without pre-store Li. The VG/CC exhibits a stable cycling performance until $450 \mathrm{~h}$ (Figure 6A). However, the voltage profile of the planar $\mathrm{Cu}$, which exhibits a fluctuant trend from $200 \mathrm{~h}$, may be due to the dendrite-induced short circuit. Figure 6B shows the voltage hysteresis profiles with cycles. The voltage hysteresis of VG/CC and planar $\mathrm{Cu}$ is reduced in the initial cycles. The voltage hysteresis of planar $\mathrm{Cu}$ rises to $35 \mathrm{mV}$ after 50 cycles and starts to fluctuate. However, the voltage hysteresis of VG gradually stabilizes at $23-25 \mathrm{mV}$ after 20 cycles. The charge transfer resistance (Rct) of VG/CC and planar $\mathrm{Cu}$ is measured by electrochemical impedance spectroscopy (EIS). An equivalent circuit model is used to simulate Rct as shown in the inset of Figure 6C. The charge transfer resistance of Li@planar $\mathrm{Cu}$ is decreased from 211.10 to $18.05 \Omega$ from the 1 st to 30 th cycle. However, Li@VG/CC is decreased from 45.04 to $11.22 \Omega$, from the 1st to 30th cycle. Li@VG/CC electrode shows faster charge transfer resistance and a more stable interface (Kolosnitsyn et al., 2011). A long-term voltage cycle with a current density of $2 \mathrm{~mA} \mathrm{~cm}^{-2}$ is also measured in Figure S7. Figure 6D also shows the long-term cycle curve at a high current density of $10 \mathrm{~mA}$ $\mathrm{cm}^{-2}$. Li@planar $\mathrm{Cu}$ undergoes a random fluctuation during the cycling because the unstable interface of $\mathrm{Li}$ on $\mathrm{Cu}$ foil. Then the voltage hysteresis drops to $\sim 92.4 \mathrm{mV}$ abruptly after two cycles due to the dendrite-induced short circuit. However, Li@VG/CC can stably cycle over 500 cycles with a smaller voltage hysteresis $(90.9 \mathrm{mV})$ and a small overpotential change. A comparison cycle performance of VG/CC with other 3D hosts is summarized in Table 1 . The cycle stability of VG/CC is one of the best compared with the other state-of-art 3D hosts.

To further prove the practical application of Li@VG/CC, LCO is used as the cathode and Li@VG/CC as the anode to fabricate a full battery. Li@VG/CC is fabricated by depositing 2 $\mathrm{mAh} \mathrm{cm}{ }^{-2}$ of lithium metal into the VG/CC host as the anode. After electrochemical deposition of Li into VG/CC host, the cell is disassembled and reassembled with LCO as the cathode. Figure 7A shows the galvanostatic charge/discharge curves of the Li@VG/CC-LCO and Li@planar Cu-LCO at 0.2 C $(1$ C = 372 
mAh $\mathrm{g}^{-1}$ ). The capacities of Li@VG/CC-LCO and Li@planar $\mathrm{Cu}-\mathrm{LCO}$ are 133 and $127 \mathrm{mAh} \mathrm{g}^{-1}$, respectively. Figure 7B is cycle performance of the Li@VG/CC-LCO and Li@planar CuLCO at 0.2 C. After 35 cycles, the capacity of Li@planar CuLCO decays rapidly down to $0 \mathrm{mAh} \mathrm{g}^{-1}$. However, Li@VG/CCLCO still maintains a capacity of $50 \mathrm{mAh} \mathrm{g}^{-1}$ at 80 cycles. Compared to planar $\mathrm{Cu}$, the capacity and the stability are significantly improved.

\section{CONCLUSIONS}

In summary, a conductive metal-free carbon-based VG/CC 3D porous structure is synthesized and used as the lithium metal host. The abundant void between VG nanosheets fully accommodates the lithium volume change and plays a significant role in inhibiting the formation of lithium dendrites. Our designed Li@VG/CC anode exhibits excellent cycle stability, high and stable Coulombic efficiency and a relatively small voltage hysteresis. Importantly, a full battery composed of Li@VG/CC as the anode and the LCO as the cathode exhibits high capacity of $133 \mathrm{mAh} \mathrm{g}^{-1}$ at $0.2 \mathrm{C}$. This special designed Li@VG/CC provides a new way as high-capacity lithium metal anode.

\section{DATA AVAILABILITY STATEMENT}

The raw data supporting the conclusions of this manuscript will be made available by the authors, without undue reservation, to any qualified researcher.

\section{REFERENCES}

Adams, B. D., Zheng, J., Ren, X., Xu, W., and Zhang, J. G. (2018). Accurate determination of Coulombic efficiency for lithium metal anodes and lithium metal batteries. Adv. Energy Mater. 8:1702097. doi: 10.1002/aenm. 201702097

Bruce, P. G., Freunberger, S. A., Hardwick, L. J., and Tarascon, J. M. (2012). $\mathrm{Li}-\mathrm{O}_{2}$ and Li-S batteries with high energy storage. Nat. Mater. 11, 19-29. doi: $10.1038 /$ nmat3191

Chen, H., Zhang, B., Zhang, J., Yu, W., Zheng, J., Ding, Z., et al. (2018). Insitu grown $\mathrm{SnS}_{2}$ nanosheets on $\mathrm{rGO}$ as an advanced anode material for lithium and sodium ion batteries. Front. Chem. 6:629. doi: 10.3389/fchem.2018. 00629

Cheng, X. B., Hou, T. Z., Zhang, R., Peng, H. J., Zhao, C. Z., Huang, J. Q., et al. (2016a). Dendrite-free lithium deposition induced by uniformly distributed lithium ions for efficient lithium metal batteries. Adv. Mater. 28, 2888-2895. doi: 10.1002/adma.201506124

Cheng, X. B., Peng, H. J., Huang, J. Q., Zhang, R., Zhao, C. Z., Zhang, Q. (2015). Dual-phase lithium metal anode containing a polysulfide-induced solid electrolyte interphase and nanostructured graphene framework for lithium-sulfur batteries. ACS Nano 9, 6373-6382. doi: 10.1021/acsnano. 5 b01990

Cheng, X. B., Yan, C., Zhang, X. Q., Liu, H., and Zhang, Q. (2018). Electronic and ionic channels in working interfaces of lithium metal anodes. ACS Energy Lett. 3, 1564-1570. doi: 10.1021/acsenergylett.8b00526

Cheng, X. B., Zhang, R., Zhao, C. Z., Wei, F., Zhang, J. G., and Zhang, Q. (2016b). A review of solid electrolyte interphases on lithium metal anode. Adv. Sci. 3:1500213. doi: 10.1002/advs.201500213

Cheng, X. B., Zhang, R., Zhao, C. Z., and Zhang, Q. (2017). Toward safe lithium metal anode in rechargeable batteries: a review. Chem. Rev. 117, 10403-10473. doi: $10.1021 /$ acs.chemrev.7b00115

\section{AUTHOR CONTRIBUTIONS}

YW developed the concept and designed the experiments. CY and CM conducted the experiments. TX, JZ, and JX built the cells and carried out the performance characterizations. YW and $\mathrm{XL}$ co-supervised the research. CK, DK, and YS revised the work critically for important intellectual content. All authors discussed the results and co-wrote the manuscript.

\section{FUNDING}

This work was supported by the National Natural Science Foundation of China (Grant Nos. 21603192, 51802288, and U1804132), China Postdoctoral Science Foundation (No. 2017M622369), Academic Improvement Program of Physics, Zhengzhou University (Grant No. 35910121), Zhengzhou University Youth Talent Startup Grant (Grant No. 32310147), the Outstanding Young Talent Research Fund of Zhengzhou University (Grant No. 1521317005), and the (Key) Project of Department of Education of Guangdong Province (Grant No. 2016KZDXM008).

\section{SUPPLEMENTARY MATERIAL}

The Supplementary Material for this article can be found online at: https://www.frontiersin.org/articles/10.3389/fchem. 2019.00714/full\#supplementary-material

Cho, H. I., Jeong, Y. C., Kim, J. H., Cho, Y. S., Kim, T., Yang, S. J., et al. (2018). Rational design of 1D partially graphitized N-doped hierarchical porous carbon with uniaxially packed carbon nanotubes for high-performance lithium-ion batteries. ACS Nano 12, 11106-11119. doi: 10.1021/acsnano.8b 05529

Chu, S., Cui, Y., and Liu, N. (2016). The path towards sustainable energy. Nat. Mater. 16, 16-22. doi: 10.1038/nmat4834

Chu, S., and Majumdar, A. (2012). Opportunities and challenges for a sustainable energy future. Nature 488, 294-303. doi: 10.1038/nature 11475

Deng, X., Liu, X., Yan, H., Wang, D., and Wan, L. (2014). Morphology and modulus evolution of graphite anode in lithium ion battery: an in situ AFM investigation. Sci. China Chem. 57, 178-183. doi: 10.1007/s11426-013-4988-4

Du, W. C., Yin, Y. X., Zeng, X. X., Shi, J. L., Zhang, S. F., Wan, L. J., et al. (2016). Wet chemistry synthesis of multidimensional nanocarbon-sulfur hybrid materials with ultrahigh sulfur loading for lithium-sulfur batteries. ACS Appl. Mater. Interfaces 8, 3584-3590. doi: 10.1021/acsami.5b07468

Ghosh, S., Polaki, S. R., Kumar, N., Amirthapandian, S., Kamruddin, M., and Ostrikov, K. (2017). Process-specific mechanisms of vertically oriented graphene growth in plasmas. Beilstein J. Nanotechnol. 8, 1658-1670. doi: 10.3762/bjnano.8.166

Grande, L., Paillard, E., Hassoun, J., Park, J. B., Lee, Y. J., Sun, Y. K., et al. (2015). The lithium/air battery: still an emerging system or a practical reality? $A d v$. Mater. 27, 784-800. doi: 10.1002/adma.201403064

Hao, Y., Wang, L., Liu, Y., Chen, H., Wang, X., Tan, C., et al. (2016). Oxygen-activated growth and bandgap tunability of large single-crystal bilayer graphene. Nat. Nanotechnol. 11:426. doi: 10.1038/nnano.2015.322

Hu, Z., Li, Z., Xia, Z., Jiang, T., Wang, G., Sun, J., et al. (2019). PECVD-derived graphene nanowall/lithium composite anodes towards highly stable lithium metal batteries. Energy Storage Mater. 22, 29-39. doi: 10.1016/j.ensm.2018. 12.020 
Huang, G., Han, J., Zhang, F., Wang, Z., Kashani, H., Watanabe, K., et al. (2019). Lithiophilic 3D nanoporous nitrogen-doped graphene for dendritefree and ultrahigh-rate lithium-metal anodes. Adv. Mater. 31:1805334. doi: 10.1002/adma.201805334

Huang, L., Wei, Q., Sun, R., and Mai, L. (2014). Nanowire electrodes for advanced lithium batteries. Front. Energy Res. 2:43. doi: 10.3389/fenrg.2014. 00043

Huang, S., Lim, Y. V., Zhang, X., Wang, Y., Zheng, Y., Kong, D., et al. (2018). Regulating the polysulfide redox conversion by iron phosphide nanocrystals for high-rate and ultrastable lithium-sulfur battery. Nano Energy 51, 340-348. doi: 10.1016/j.nanoen.2018.06.052

Jiang, Z., Xu, T., Dai, S., Yan, C., Ma, C., Wang, X., et al. (2019). 3D mesoporous $\mathrm{Ni}(\mathrm{OH})_{2} / \mathrm{WS}_{2}$ nanofibers with highly enhanced performances for hybrid supercapacitors. Energy Technol. 7:1800476. doi: 10.1002/ente.2018 00476

Kolosnitsyn, V. S., Kuzmina, E. V., Karaseva, E. V., and Mochalov, S. E. (2011). A study of the electrochemical processes in lithium-sulphur cells by impedance spectroscopy. J. Power Sources 196, 1478-1482. doi: 10.1016/j.jpowsour.2010.08.105

Kong, D., Wang, Y., Huang, S., Lim, Y. V., Zhang, J., Sun, L., et al. (2019). Surface modification of $\mathrm{Na}_{2} \mathrm{Ti}_{3} \mathrm{O}_{7}$ nanofibre arrays using $\mathrm{N}$-doped graphene quantum dots as advanced anodes for sodium-ion batteries with ultra-stable and high-rate capability. J. Mater. Chem. A 7, 12751-12762. doi: 10.1039/C9TA 01641D

Li, H., Zhang, B., Wang, X., Zhang, J., An, T., Ding, Z., et al. (2019). Heterostructured $\mathrm{SnO}_{2}-\mathrm{SnS}_{2} @ \mathrm{C}$ embedded in nitrogen-doped graphene as a robust anode material for lithium-ion batteries. Front. Chem. 7:339. doi: $10.3389 /$ fchem.2019.00339

Li, Q., Zhu, S., and Lu, Y. (2017). 3D porous Cu current collector/Li-metal composite anode for stable lithium-metal batteries. Adv. Funct. Mater. 27:1606422. doi: 10.1002/adfm.201606422

Li, Y., Huang, W., Li, Y., Pei, A., Boyle, D. T., and Cui, Y. (2018a). Correlating structure and function of battery interphases at atomic resolution using cryoelectron microscopy. Joule 2, 2167-2177. doi: 10.1016/j.joule.2018. 08.004

Li, Y., Shi, Z., Lei, L., Ma, Z., Zhang, F., Li, S., et al. (2018b). Controllable vapor-phase growth of inorganic perovskite microwire networks for highefficiency and temperature-stable photodetectors. ACS Photonics 5, 2524-2532. doi: 10.1021 /acsphotonics.8b00348

Lim, Y. V., Huang, S., Hu, J., Kong, D., Wang, Y., Xu, T., et al. (2019a). Explicating the sodium storage kinetics and redox mechanism of highly pseudocapacitive binary transition metal sulfide via operando techniques and abinitio evaluation. Small Methods 3:1900112. doi: 10.1002/smtd.201900112

Lim, Y. V., Huang, S., Wu, Q., Zhang, Y., Kong, D., Wang, Y., et al. (2019b). Rhenium disulfide nanosheets/carbon composite as novel anodes for highrate and long lifespan sodium-ion batteries. Nano Energy 61, 626-636. doi: 10.1016/j.nanoen.2019.04.041

Lin, D., Liu, Y., and Cui, Y. (2017). Reviving the lithium metal anode for high-energy batteries. Nat. Nanotechnol. 12, 194-206. doi: 10.1038/nnano. 2017.16

Lin, D., Liu, Y., Liang, Z., Lee, H. W., Sun, J., Wang, H., et al. (2016). Layered reduced graphene oxide with nanoscale interlayer gaps as a stable host for lithium metal anodes. Nat. Nanotechnol. 11, 626-632. doi: 10.1038/nnano.2016.32

Liu, B., Zhang, J. G., and Xu, W. (2018). Advancing lithium metal batteries. Joule 2, 833-845. doi: 10.1016/j.joule.2018.03.008

Liu, F., Xu, R., Hu, Z., Ye, S., Zeng, S., Yao, Y., et al. (2019). Regulating lithium nucleation via CNTs modifying carbon cloth film for stable Li metal anode. Small 15:1803734. doi: 10.1002/smll.201803734

Liu, K., Li, Z., Xie, W., Li, J., Rao, D., Shao, M., et al. (2018). Oxygen-rich carbon nanotube networks for enhanced lithium metal anode. Energy Storage Mater. 15, 308-314. doi: 10.1016/j.ensm.2018.05.025

Liu, L., Yin, Y. X., Li, J. Y., Wang, S. H., Guo, Y. G., and Wan, L. J. (2018). Uniform lithium nucleation/growth induced by lightweight nitrogen-doped graphitic carbon foams for high-performance lithium metal anodes. Adv. Mater. 30:1706216. doi: 10.1002/adma.2017 06216
Liu, S., Wang, A., Li, Q., Wu, J., Chiou, K., Huang, J., et al. (2018). Crumpled graphene balls stabilized dendrite-free lithium metal anodes. Joule 2, 184-193. doi: 10.1016/j.joule.2017.11.004

Liu, Y., Qin, X., Zhang, S., Zhang, L., Kang, F., Chen, G., et al. (2019). A scalable slurry process to fabricate a $3 \mathrm{D}$ lithiophilic and conductive framework for a high performance lithium metal anode. J. Mater. Chem. A 7, 13225-13233. doi: 10.1039/C9TA01929D

Ma, C., Xu, T., and Wang, Y. (in press). Advanced carbon nanostructures for future high performance sodium metal anodes. Energy Storage Mater. doi: 10.1016/j.ensm.2019.09.007

Ma, Y., Yao, B., Zhang, M., Bai, H., and Shi, G. (2018). Inhibiting the growth of lithium dendrites at high current densities with oriented graphene foam. $J$. Mater. Chem. A 6, 15603-15609. doi: 10.1039/C8TA04911D

Ma, Z., Yuan, X., Li, L., Ma, Z. F., Wilkinson, D. P., Zhang, L., et al. (2015). A review of cathode materials and structures for rechargeable lithium-air batteries. Energy Environ. Sci. 8, 2144-2198. doi: 10.1039/C5EE 00838G

Manthiram, A., Chung, S. H., and Zu, C. (2015). Lithium-sulfur batteries: progress and prospects. Adv. Mater. 27, 1980-2006. doi: 10.1002/adma.201405115

Meng, Q., Deng, B., Zhang, H., Wang, B., Zhang, W., Wen, Y., et al. (2019). Heterogeneous nucleation and growth of electrodeposited lithium metal on the basal plane of single-layer graphene. Energy Storage Mater. 16, 419-425. doi: 10.1016/j.ensm.2018.06.024

Ren, F., Lu, Z., Zhang, H., Huai, L., Chen, X., Wu, S., et al. (2018). Pseudocapacitance induced uniform plating/stripping of $\mathrm{Li}$ metal anode in vertical graphene nanowalls. Adv. Funct. Mater. 28:1805638. doi: 10.1002/adfm.201805638

Tian, H., Seh, Z. W., Yan, K., Fu, Z., Tang, P., Lu, Y., et al. (2017). Theoretical investigation of $2 \mathrm{D}$ layered materials as protective films for lithium and sodium metal anodes. Adv. Energy Mater. 7:1602528. doi: 10.1002/aenm.201602528

Tian, R., Duan, H., Guo, Y., Li, H., and Liu, H. (2018a). High-Coulombic-efficiency carbon/Li clusters composite anode without precycling or prelithiation. Small 14:1802226. doi: 10.1002/smll.201802226

Tian, R., Wan, S., Guan, L., Duan, H., Guo, Y., Li, H., et al. (2018b). Oriented growth of Li metal for stable $\mathrm{Li} /$ carbon composite negative electrode. Electrochim. Acta 292, 227-233. doi: 10.1016/j.electacta.2018.09.165

Wang, H., Wang, C., Matios, E., and Li, W. (2017). Critical role of ultrathin graphene films with tunable thickness in enabling highly stable sodium metal anodes. Nano Lett. 17, 6808-6815. doi: 10.1021/acs.nanolett.7b03071

Wang, T. S., Liu, Y., Lu, Y. X., Hu, Y. S., and Fan, L. Z. (2018a). Dendrite-free Na metal plating/stripping onto 3D porous Cu hosts. Energy Storage Mater. 15, 274-281. doi: 10.1016/j.ensm.2018.05.016

Wang, Y., Chen, B., Seo, D. H., Han, Z. J., Wong, J. I., Ostrikov, K., et al. (2016). $\mathrm{MoS}_{2}$-coated vertical graphene nanosheet for high-performance rechargeable lithium-ion batteries and hydrogen production. NPG Asia Mater. 8:e268. doi: 10.1038/am.2016.44

Wang, Y., Kong, D., Huang, S., Shi, Y., Ding, M., Von Lim, Y., et al. (2018b). 3D carbon foam-supported $\mathrm{WS}_{2}$ nanosheets for cable-shaped flexible sodium ion batteries. J. Mater. Chem. A 6, 10813-10824. doi: 10.1039/C8TA02773K

Wu, D., Wang, Y., Zeng, L., Jia, C., Wu, E., Xu, T., et al. (2018). Design of $2 \mathrm{D}$ layered $\mathrm{PtSe}_{2}$ heterojunction for the high-performance, roomtemperature, broadband, infrared photodetector. ACS Photonics 5, 3820-3827. doi: 10.1021 /acsphotonics.8b00853

Xu, J., Tang, H., Xu, T., Wu, D., Shi, Z., Tian, Y., et al. (2017). Porous NiO hollow quasi-nanospheres derived from a new metal-organic framework template as high-performance anode materials for lithium ion batteries. Ionics 23, 3273-3280. doi: 10.1007/s11581-017-2160-4

Xu, W., Wang, J., Ding, F., Chen, X., Nasybulin, E., Zhang, Y., et al. (2014). Lithium metal anodes for rechargeable batteries. Energy Environ. Sci. 7, 513-537. doi: 10.1039/C3EE40795K

Yan, K., Lu, Z., Lee, H. W., Xiong, F., Hsu, P. C., Li, Y., et al. (2016). Selective deposition and stable encapsulation of lithium through heterogeneous seeded growth. Nat. Energy 1:16010. doi: 10.1038/nenergy.2016.10

Zhang, K., Lee, G. H., Park, M., Li, W., and Kang, Y. M. (2016). Recent developments of the lithium metal anode for rechargeable nonaqueous batteries. Adv. Energy Mater. 6:1600811. doi: 10.1002/aenm. 201600811 
Zhang, Y., Liu, B., Hitz, E., Luo, W., Yao, Y., Li, Y., et al. (2017). A carbon-based 3D current collector with surface protection for Li metal anode. Nano Res. 10, 1356-1365. doi: 10.1007/s12274-017-1461-2

Zhang, Y. J., Liu, S. F., Wang, X. L., Zhong, Y., Xia, X. H., Wu, J. B., et al. (2018). Composite Li metal anode with vertical graphene host for high performance Li-S batteries. J. Power Sources 374, 205-210. doi: 10.1016/j.jpowsour.2017. 10.057

Zuo, T. T., Wu, X. W., Yang, C. P., Yin, Y. X., Ye, H., Li, N. W., et al. (2017). Graphitized carbon fibers as multifunctional 3D current collectors for high areal capacity Li anodes. Adv. Mater. 29:1700389. doi: 10.1002/adma. 201700389
Conflict of Interest: The authors declare that the research was conducted in the absence of any commercial or financial relationships that could be construed as a potential conflict of interest.

Copyright (c) 2019 Yan, Xu, Ma, Zang, Xu, Shi, Kong, Ke, Li and Wang. This is an open-access article distributed under the terms of the Creative Commons Attribution License (CC BY). The use, distribution or reproduction in other forums is permitted, provided the original author(s) and the copyright owner(s) are credited and that the original publication in this journal is cited, in accordance with accepted academic practice. No use, distribution or reproduction is permitted which does not comply with these terms. 